\title{
SubMo-GNN: Property- and Structure-Aware Diverse Molecular Selection with Representation Learning and Mathematical Diverse-Selection Framework
}

\section{Tomohiro Nakamura}

The University of Tokyo

Shinsaku Sakaue ( $\nabla$ sakaue@mist.i.u-tokyo.ac.jp )

The University of Tokyo

Kaito Fujii

National Institute of Informatics

Yu Harabuchi

Hokkaido University

Satoshi Maeda

Hokkaido University

Satoru Iwata

The University of Tokyo

\section{Research Article}

Keywords: SubMo-GNN, molecules, chemical space, graph neural networks (GNNs)

Posted Date: September 2nd, 2021

DOl: https://doi.org/10.21203/rs.3.rs-849372/v1

License: (9) (i) This work is licensed under a Creative Commons Attribution 4.0 International License.

Read Full License 


\title{
SubMo-GNN: Property- and Structure-Aware Diverse Molecular Selection with Representation Learning and Mathematical Diverse-Selection Framework
}

\author{
Tomohiro Nakamura ${ }^{1,6}$, Shinsaku Sakaue ${ }^{1,6, *}$, Kaito Fujii ${ }^{2,6, *}$, Yu Harabuchi ${ }^{3,4,6, *}$, Satoshi \\ Maeda $^{3,5,4,6}$, and Satoru Iwata ${ }^{1,4,6}$
}

\author{
${ }^{1}$ The University of Tokyo, Department of Mathematical Informatics, Hongo 7-3-1, Bunkyo-ku, Tokyo 113-8656, \\ Japan. \\ ${ }^{2}$ National Institute of Informatics, Hitotsubashi 2-1-2, Chiyoda-ku, Tokyo 101-8430, Japan. \\ ${ }^{3}$ Hokkaido University, Department of Chemistry, Faculty of Science, Kita 10 Nishi 8, Kita-ku, Sapporo, Hokkaido \\ 060-0810, Japan. \\ ${ }^{4}$ Institute for Chemical Reaction Design and Discovery (WPI-ICReDD), Hokkaido University, Kita 21 Nishi 10, \\ Kita-ku, Sapporo, Hokkaido 001-0021, Japan. \\ ${ }^{5}$ National Institute for Materials Science (NIMS), Research and Services Division of Materials Data and Integrated \\ System (MaDIS), Tsukuba, Ibaraki 305-0044, Japan. \\ ${ }^{6}$ JST, ERATO Maeda Artificial Intelligence for Chemical Reaction Design and Discovery Project, Kita 10 Nishi 8, \\ Kita-ku, Sapporo, Hokkaido 060-0810, Japan. \\ *sakaue@mist.i.u-tokyo.ac.jp; fujiik@nii.ac.jp; y_harabuchi@sci.hokudai.ac.jp
}

\section{ABSTRACT}

Selecting diverse molecules from unexplored areas of chemical space is one of the most important tasks for discovering novel molecules and reactions. This paper develops a new method for selecting a diverse subset of molecules from a given molecular list by utilizing two techniques studied in machine learning and mathematical optimization: graph neural networks (GNNs) for learning vector representation of molecules and a diverse-selection framework called submodular function maximization. Our method first trains a GNN with property prediction tasks, and then the trained GNN transforms molecular graphs into molecular vectors, which capture both properties and structures of molecules. Finally, to obtain a diverse subset of molecules, we define a submodular function, which quantifies the diversity of molecular vectors, and find a subset of molecular vectors with a large submodular function value. This can be done efficiently by using the greedy algorithm, and the diversity of selected molecules measured by the submodular function value is mathematically guaranteed to be at least $63 \%$ of that of an optimal selection. We also introduce a new evaluation criterion to measure the diversity of selected molecules based on molecular properties. Computational experiments confirm that our method successfully selects diverse molecules from the QM9 dataset regarding the property-based criterion, while performing comparably to existing methods regarding a standard structure-based criterion. The proposed method enables researchers to obtain diverse sets of molecules for discovering new molecules and novel chemical reactions, and the proposed diversity criterion is useful for discussing the diversity of molecular libraries from a new property-based perspective.

\section{Introduction}

Chemical space, ${ }^{1-4}$ a concept to represent an ensemble of chemical species, was originally established in medicinal chemistry ${ }^{2,5}$ and is used in a wide area of chemistry. The size of chemical space, i.e., the number of molecules in it, is estimated to be $10^{60}$ even if it is limited to drug-like molecules, ${ }^{6}$ and other estimations of chemical-space sizes have also been reported..$^{4,7}$ In any case, the number of molecules is too large to explore exhaustively. Currently, more than 68 million molecules are registered in the chemical abstracts service (CAS) of Americal Chemical Society, ${ }^{8,9}$ and some accessible online molecular databases, e.g., PubChem, ${ }^{10}$ ZINC, ${ }^{11}$ have been constructed. Moreover, owing to recent advances in high throughput screening, chemoinformatics, ${ }^{12}$ and machine learning, ${ }^{13}$ many chemical compounds have been discovered from chemical space in the fields of organic light-emitting diode, ${ }^{14}$ organic synthesis, ${ }^{15}$ and catalyst. ${ }^{16}$ These are, however, small fractions of chemical space, and vast areas remain unexplored.

Selecting diverse molecules from chemical space is an important task for discovering molecules that exhibit novel properties and new chemical reactions. ${ }^{3,17}$ In medicinal chemistry, diversity selection algorithms have been widely studied for exploring chemical space and discovering bioactive molecules. ${ }^{5,18-21}$ The diversity of a set of molecules is also essential in molecular 
library design. ${ }^{17,22}$ Furthermore, when analyzing the quality of molecular libraries, the way to assess their diversity is crucial. This paper contributes to diverse molecular selection by proposing a novel selection framework and a new criterion for evaluating the diversity of molecules.

For computing the diversity of sets of molecules, most existing methods start by specifying molecular descriptors, which encode molecules as vectors. Examples of molecular descriptors include ECFP, ${ }^{23}$ MACCS keys, ${ }^{24}$ and Daylight fingerprints, ${ }^{25}$ which typically encode structural information of molecules as binary vectors. Given such descriptors, pairwise dissimilarities are defined to quantify how dissimilar two molecules are. A widely used pairwise dissimilarity is the Tanimoto coefficient (more precisely, the Tanimoto coefficient indicates a similarity value, and subtracting it from 1 yields the dissimilarity). ${ }^{26}$ Computation of molecular similarity values constitutes a broad research area, and other approaches such as a 3D-structure-based method have also been developed. ${ }^{27}$ With such pairwise dissimilarity measures, the diversity of sets of molecules is evaluated based on, e.g., the mean pairwise dissimilarity (MPD) or the mean distance to closest neighbors calculated over selected molecules.

While the diversity of molecules can be computed as above, selecting molecules that maximize a diversity measure from given molecular lists is computationally more challenging. For example, a naive brute force search for selecting 10 out of 100 compounds requires calculating diversity values $\left(\begin{array}{c}100 \\ 10\end{array}\right)$ times. To overcome this computational difficulty, the greedy algorithm, which iteratively selects a new molecule that is the most dissimilar to a set of currently selected molecules, has been widely used as an efficient heuristic method. ${ }^{28}$ In each iteration, the dissimilarity between a new molecule and a set of selected molecules is computed according to a certain rule, e.g., MaxSum ${ }^{29}$ and MaxMin, ${ }^{30,31}$ and the choice of such rules affects outputs of the greedy algorithm. The diversity of molecular sets obtained by the greedy algorithm is usually evaluated with, e.g., the MPD defined with the Tanimoto coefficient of MACCS keys. Thus calculated diversity values intrinsically depend on the choice of molecular descriptors and pairwise dissimilarities. Consequently, the existing framework for selecting molecules and evaluating the diversity puts much weight on structural information of molecules since molecular descriptors usually encode structural information of molecules and pairwise dissimilarities are calculated based on such structure-based descriptors.

On the other hand, exploration of chemical space that takes the diversity of molecular properties into account has been reported to be effective for discovering novel functional materials. ${ }^{32}$ Also, when it comes to discovering novel reactions, examining collections of molecules that are diverse regarding molecular properties (in particular, reactivity) is vital for efficient exploration. Therefore, utilizing not only structural information but also properties of molecules can be a promising approach to pushing the diverse molecular selection framework to the next level, facilitating the discovery of novel molecules and new chemical reactions.

In the field of machine learning, neural network $(\mathrm{NN})$ architectures have yielded great success in various areas such as image recognition and natural language processing. Following the achievements, researchers have applied them to molecular property prediction tasks. Among such approaches, graph neural networks (GNNs) have been gaining attention since many GNN-based prediction methods have achieved high performances. ${ }^{33-38}$ GNNs transform molecular graphs into vectors, which are used in downstream property prediction tasks. Notably, GNNs generate vectors taking both molecular properties and structural information of molecules into account, and it is reported that molecular vectors obtained from trained GNNs successfully reflect chemists' intuition of molecular structures. ${ }^{36}$ Therefore, GNN-based molecular vectors can be effective alternatives to the aforementioned traditional molecular descriptors. However, to leverage GNN-based vectors for selecting diverse molecules, we need to discuss how to select diverse molecular vectors generated by GNNs, for which the existing structure-based selection framework is not necessarily appropriate.

Mathematically, the problem of selecting diverse items (in our case, molecular vectors) has been widely studied as submodular function maximization..$^{39,40}$ This framework is one of the best ways for diverse selection due to the following two advantages. First, many submodular functions for quantifying the diversity have been developed in various fields, and thus we can choose an appropriate one to achieve desirable diverse selection. In particular, some submodular functions can represent relationships between multiple molecules that pairwise similarities cannot capture. Such functions can offer us the potential for going beyond the existing pairwise-similarity-based framework. Second, and more importantly, we can mathematically guarantee the greedy algorithm to select near-optimally diverse molecules in terms of submodular function values. Specifically, resulting submodular function values are guaranteed to be at least $63 \%$ of those achieved by optimal selection. ${ }^{39}$ Moreover, the empirical performance of the greedy algorithm for submodular function maximization is known to be much better; it often achieves more than $90 \%$ of optimal values. Therefore, the submodularity-based approach enables us to efficiently obtain near-optimally diverse sets of molecules without relying on costly selection algorithms such as the brute force search.

This paper proposes a new diverse molecular selection method by utilizing the aforementioned GNN-based molecular vectors and the submodularity-based selection method. First, we train a GNN with property prediction tasks and use the trained GNN to transform molecular graphs into molecular vectors. Then, we define a submodular function that quantifies the diversity of those molecular vectors as volumes of parallelotopes spanned by them. Owing to the submodularity of the function, we can select near-optimally diverse molecular vectors by using the greedy algorithm. Both GNNs and submodular function maximization are known to be effective in various tasks, and thus each of them has been well studied. However, few existing 
(a) Step 1: Training

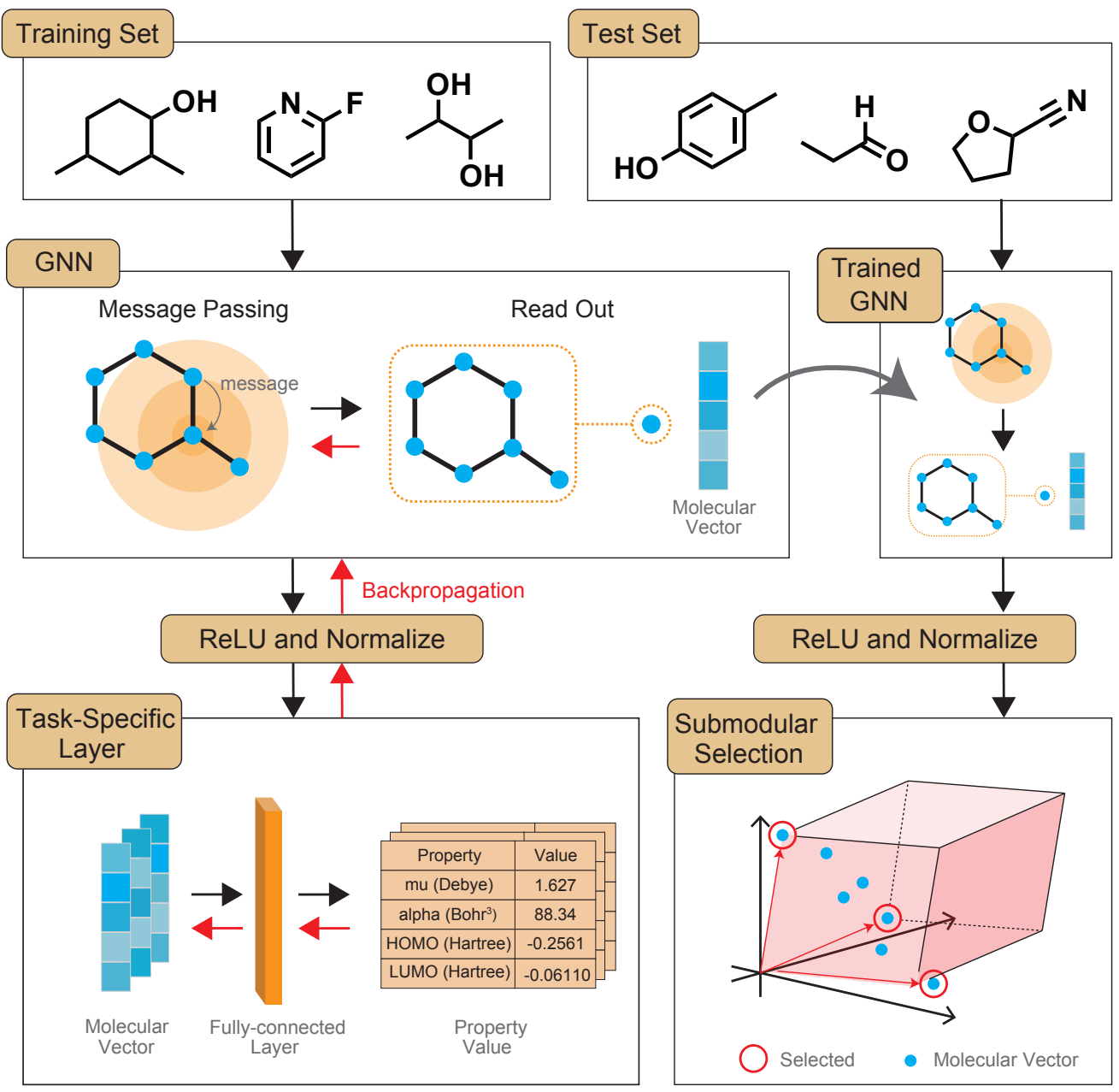

Figure 1. A high-level sketch of our method. In Step 1, a GNN is trained with property prediction tasks. The black and red arrows indicate the forward pass and backpropagation, respectively. In Step 2, the trained GNN is used to generate molecular vectors of candidate molecules, and then molecules are selected via submodular function maximization.

studies utilize both of them for a single purpose. The only exception is a recent study on multi-robot action selection, ${ }^{41}$ which uses GNNs in selection methods, while we use GNNs to design submodular functions. In view of this, our work provides a new type of application combining GNNs and submodular function maximization. Furthermore, to evaluate the diversity of selected molecules based on molecular property values, we introduce a new diversity measure using the Wasserstein distance ${ }^{42,43}$ to uniform distributions defined on molecular property values. This property-based measure can play a complementary role to the existing structure-based measures such as the MPD of the Tanimoto coefficients, thus enabling researchers to more profoundly discuss the diversity of molecules. Computational experiments compare the proposed method with the existing structure-based methods and confirm that our method selects more diverse molecules regarding molecular properties. Furthermore, although our method does not explicitly use structure-based descriptors (e.g., ECFP and MACCS key), it successfully selects diverse molecules in terms of MPD values calculated with the Tanimoto coefficient of such structure-based descriptors.

\section{Method}

This section presents our molecular selection method, which comprises two steps: training a GNN that generates molecular vectors and selecting GNN-based molecular vectors via submodular function maximization.

Figure 1 shows a high-level sketch of our method. In Step 1, we train a GNN and task-specific layers with property 
prediction tasks, where the GNN converts molecular graphs into molecular vectors and the task-specific layers take them as input and predict molecular properties. In this step, parameters of the GNN and the task-specific layers are updated by the error backpropagation method. In Step 2, we transform graphs of candidate molecules into molecular vectors by using the GNN trained in Step 1, and then select a predetermined number of molecular vectors based on submodular function maximization.

We also introduce a new property-based diversity criterion, which quantifies the diversity of selected molecules as the Wasserstein distance to uniform distributions defined on molecular property values. Intuitively, we regard a set of molecules as diverse if the property values of those molecules are evenly distributed.

\section{Graph neural networks for generating molecular vectors}

We briefly explain how molecular vectors are generated by GNNs, which are deep learning architectures that work on graph domains. Taking a graph with node and edge features as input, GNNs capture structures of the graph by iteratively passing messages, which are calculated based on the features. Specifically, each node iteratively receives messages from its neighbors, aggregates them, and pass them to its neighbors; after this message passing phase, a molecular vector, denoted by $\boldsymbol{x}$, is computed based on the resulting messages of all nodes. Along the way, messages are updated with certain parameterized functions. Our specific choice of a GNN architecture is Attentive FP, ${ }^{36}$ which is reported to achieve high performances in molecular property prediction. For the sake of completeness, we present mathematical details of GNNs in the supplementary information.

In the task-specific layer, molecular properties, $\boldsymbol{y}$, are predicted with molecular vector $\boldsymbol{x}$ via simple linear regression as $\hat{\boldsymbol{y}}=\boldsymbol{W} \boldsymbol{x}+\boldsymbol{b}$, where $\hat{\boldsymbol{y}}$ is a prediction of $\boldsymbol{y}$. In the training step (Step 1 in Figure 1), with a loss function defined by the mean square error between $\hat{\boldsymbol{y}}$ and $\boldsymbol{y}$, we update $\boldsymbol{W}, \boldsymbol{b}$, and the parameters of the GNN by backpropagation. Consequently, the GNN, which can capture structures of molecular graphs via message passing, is trained to predict molecular properties. Therefore, the trained GNN generates molecular vectors taking both structures and properties of molecules into account.

\section{Selection of diverse molecular vectors}

Given molecular vectors generated by the trained GNN, we aim to obtain a set of diverse molecules by selecting diverse molecular vectors. For selecting diverse vectors, we utilize the mathematical framework called submodular function maximization.

\section{Submodular function maximization}

Submodular function maximization has been studied in the field of combinatorial optimization. This framework enables development of effective diverse selection methods by offering flexible models for representing the diversity and efficient selection algorithms with mathematical performance guarantees; below we detail these two advantages.

The first advantage of using the submodular-function-maximization framework is that there are various known functions for representing the diversity. To find a diverse subset from a large pool of molecules, researchers usually specify a diversity criterion and search for a diverse subset based on the criterion. Here, a diversity criterion is formally regarded as a set function, which assigns to each subset a real value that indicates how diverse the subset is. Some of such functions have a special property called submodularity, and they are called submodular functions. Many submodular functions have been developed as diversity criteria for various kinds of data such as images, documents, and videos. Therefore, we can choose a suitable function from them for modeling the diversity of molecular vectors. For example, the Shannon entropy is known to satisfy submodularity with respect to the selection of random variables. Other diversity criteria that have submodularity include the ROUGE-N score for document summarization ${ }^{44,45}$ and facility location functions. ${ }^{46}$ In the area of bioinformatics, submodular functions for peptide identification are also developed. ${ }^{47}$

The second advantage of the submodular-function-maximization framework is that we can utilize various simple, efficient, and mathematically rigorous algorithms for selecting a diverse subset. When selecting a subset from a large number of molecular vectors, there are exponentially many possible candidate subsets. Therefore, we need efficient algorithms for finding diverse subsets. In a series of studies on submodular function maximization, many simple and efficient algorithms for finding subsets with large submodular function values have been developed. Notably, the resulting submodular function values are often guaranteed to be nearly optimal by mathematical analyses. Therefore, once we specify a submodular function as a diversity criterion, we can automatically ensure that those algorithms return highly diverse subsets with respect to the criterion. Among such algorithms, the greedy algorithm is widely used due to its simplicity, efficiency, and strong mathematical guarantee. ${ }^{39}$

In the supplementary information, we present mathematical details of submodular function maximization and the greedy algorithm.

\section{Log-determinant function}

In our computational experiments, we use a submodular function called a log-determinant function, which quantifies the diversity of selected molecular vectors based on the volume of a parallelotope spanned by the selected vectors. As depicted in Figure $2 \mathrm{a}$, the more diverse the directions of vectors are, the larger the volume of the parallelotope spanned by them. Thus the log-determinant function provides a volume-based measure of the diversity of vectors, and it is often used for expressing 


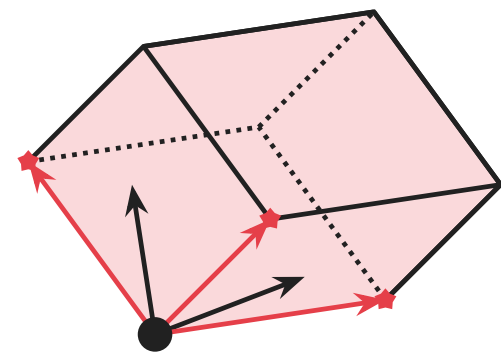

(a)

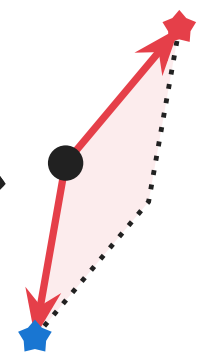

(b)

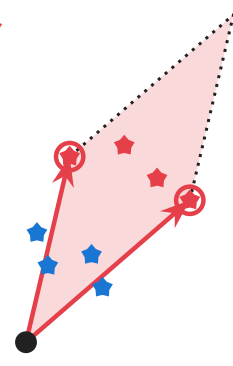

(c)

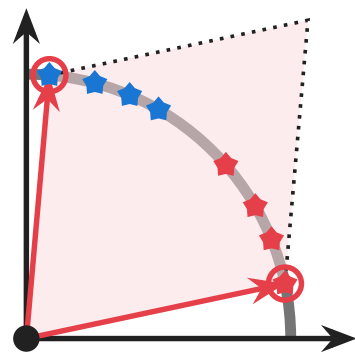

(d)

Figure 2. Graphical explanation of (a) the log-determinant function, (b) the effect of ReLU, and (c and d) the effect of normalization. In all figures above, the black dot indicates the origin. Figure $2 \mathrm{a}$ is a parallelotope spanned by vectors colored in red. Figure $2 b$ illustrates an example where the log-determinant function value for dissimilar vectors becomes small if vectors are allowed to have negative elements. Here and in the next two figures, points with different colors (red and blue) represent molecules with dissimilar properties, while those with the same colors have similar properties. Figure $2 \mathrm{c}$ shows why maximizing the log-determinant function without normalization may result in a non-diverse selection, and Figure $2 \mathrm{~d}$ presents how normalization helps the log-determinant function maximization to select diverse vectors.

the diversity of vector datasets. ${ }^{48}$ Note that the volume-based diversity can capture relationships of vectors that cannot be represented in a pairwise manner. Therefore, the log-determinant function yields a different selection rule than MaxSum and MaxMin.

Formally, the log-determinant function is defined as follows. Suppose that $n$ candidate molecules are numbered by $1, \ldots, n$ and that the $i$-th molecule is associated with $m$-dimensional molecular vector $\boldsymbol{x}_{i}$ for $i=1, \ldots, n$. Let $\boldsymbol{X}=\left[\boldsymbol{x}_{1} \boldsymbol{x}_{2} \ldots \boldsymbol{x}_{n}\right]$ be an $m \times n$ matrix whose columns are given by $n$ molecular vectors. For any $S \subseteq N:=\{1, \ldots, n\}$, we denote by $\boldsymbol{X}[S]$ an $m \times|S|$ submatrix of $\boldsymbol{X}$ with columns restricted to $S$. We define the log-determinant function $f_{\text {logdet }}$ by

$$
f_{\text {logdet }}(S)=\log \operatorname{det}\left(\boldsymbol{X}[S]^{\top} \boldsymbol{X}[S]+\mathbf{I}_{|S|}\right)
$$

for any $S \subseteq N$, where $\mathbf{I}_{|S|}$ is the $|S| \times|S|$ identity matrix. The relationship between the $f_{\text {logdet }}$ value and the volume of a parallelotope can be formally described as follows. Let $\tilde{\boldsymbol{x}}_{i}(i=1, \ldots, n)$ be a vector of length $m+n$ such that the first $m$ elements are given by $\boldsymbol{x}_{i}$, the $(m+i)$-th element is 1 , and the others are 0 . When $S \subseteq N$ is selected, $f_{\text {logdet }}(S)$ indicates the volume of a parallelotope spanned by $\left\{\tilde{\boldsymbol{x}}_{i}\right\}_{i \in S}$.

Given the function, $f_{\text {logdet }}$, and the number, $k$, of molecules to be selected, the greedy algorithm operates as follows: it first sets $S=\emptyset$ and sequentially adds $i \in N \backslash S$ with the largest $f_{\text {logdet }}(S \cup\{i\})-f_{\text {logdet }}(S)$ value to $S$ while $|S|<k$ holds. In our computational experiments, we use a fast implementation of the greedy algorithm specialized for the log-determinant function. ${ }^{49}$ Function $f_{\text {logdet }}$ satisfies $f_{\text {logdet }}(\emptyset)=0$, monotonicity (i.e., $S \subseteq T$ implies $f_{\text {logdet }}(S) \leq f_{\text {logdet }}(T)$ ), and submodularity. With these properties, we can mathematically guarantee that the greedy algorithm returns a subset whose $f_{\text {logdet }}$ value is at least $1-1 / \mathrm{e} \approx 63 \%$ of an optimal selection.

\section{Refinements to molecular vector generation: ReLU and normalization}

We refine the GNN-based vector generation process so that it works better with the log-determinant function. Specifically, we make GNNs output non-negative and normalized. Below we detail why we need these refinements and explain how to modify the vector generation process.

First, as in Figure $2 \mathrm{~b}$, if vectors are allowed to have negative elements, nearly origin-symmetric vectors form a parallelotope with a small volume even though their directions are diverse. Consequently, the log-determinant function fails to indicate that such molecular vectors correspond to diverse molecules. To circumvent this issue, we add a ReLU layer to the end of the GNN, which makes all entries of output vectors non-negative.

Second, if GNNs are allowed to output vectors with different norms, task-specific layers may distinguish molecules with different properties based on the norm of molecular vectors. In such cases, maximizing the log-determinant function may result in selecting non-diverse vectors due to the following reason. As mentioned above, the log-determinant function represents the volume of the parallelotope spanned by selected vectors, and the volume becomes larger if selected vectors have larger norms. Consequently, molecular vectors with larger norms are more likely to be selected, which may result in selecting molecules with almost the same properties as in Figure 2c. To resolve this problem, after applying the ReLU layer, we normalize molecular vectors so that their norms become 1 by projecting them onto a hypersphere. In other words, we add a layer that transforms 


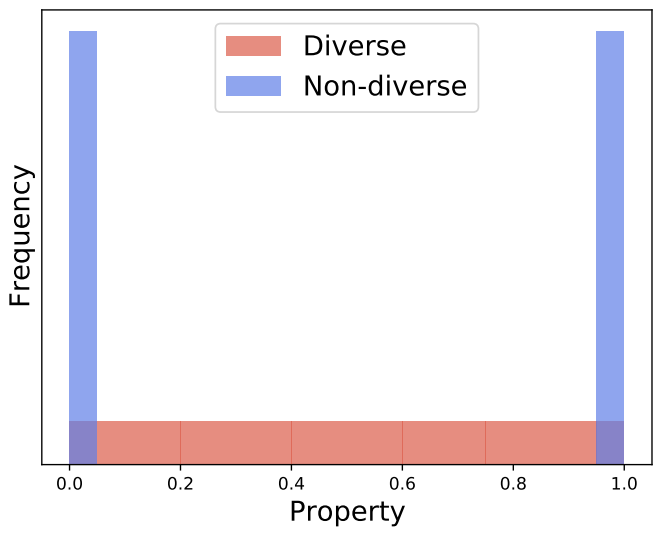

(a)

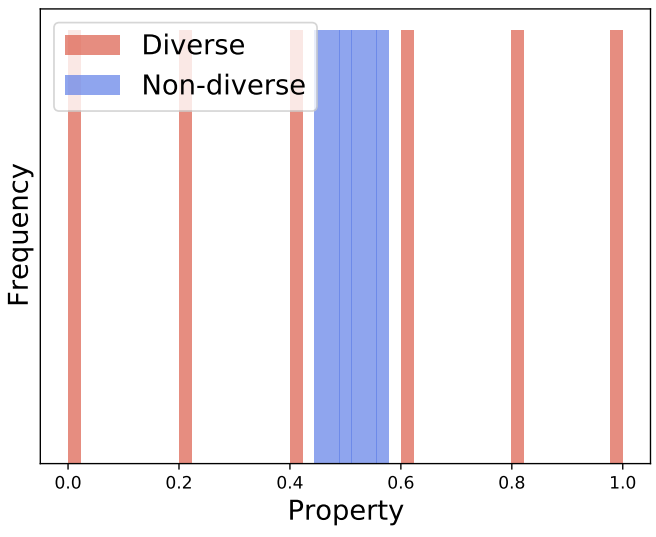

(b)

Figure 3. Graphical explanation of why the (a) variance and (b) Kullback-Leibler (KL) divergence are inappropriate. In Figure 3a, the variance of a diverse set (red) is smaller than that of a non-diverse set (blue), which does not suit our idea of diversity. In Figure 3b, the KL divergence of a diverse set (red) is equal to that of a non-diverse set (blue).

molecular vector $\boldsymbol{x}$ as

$$
\hat{x}=\frac{x}{\|x\|} .
$$

As a result, $\hat{\boldsymbol{x}}$ becomes non-negative and its norm is equal to 1 . In the training phase, we train the GNN with the additional ReLU and normalization layers, where non-negative normalized vector $\hat{\boldsymbol{x}}$ is used for predicting property values as $\hat{\boldsymbol{y}}=\boldsymbol{W} \hat{\boldsymbol{x}}+\boldsymbol{b}$. Due to the above normalization, the task-specific layers cannot distinguish molecular vectors by using their norms, and thus the GNN learns to generate molecular vectors so that task-specific layers can predict molecular property values based not on norms but on angles of vectors. Consequently, as illustrated in Figure 2d, diverse molecular vectors can be obtained by maximizing the log-determinant function value.

\section{Property-based evaluation of diversity}

By using our selection method, we can select molecules so that corresponding molecular vectors are diverse. However, even if molecular vectors are diverse, selected molecules themselves may not be diverse. This issue is also the case with the existing structure-based methods, and it has been overlooked in previous studies. That is, the existing methods select molecules that are diverse in terms of the Tanimoto coefficient of molecular descriptors (e.g., MACCS keys or ECFP), and thus it is natural that those methods can achieve high mean parwise distance (MPD) values, which are also calculated by using the Tanimoto coefficient of such descriptors. If we are to evaluate selection methods fairly, we need diversity criteria that do not use molecular descriptors employed by selection methods. This section presents such a criterion for evaluating the diversity of selected molecules in terms of their property values without using molecular vectors. In contrast to the existing structure-based criteria (e.g., the aforementioned MPD values), our criterion is based on the diversity of property values, thus offering a new perspective for evaluating the diversity of molecules.

Our idea is to regard molecular property values as diverse if evenly distributed over an interval on the property-value line. We quantify this notion of the diversity using a statistical distance between a distribution of property values of selected molecules and a uniform distribution. As a distance between two distributions, we use the Wasserstein distance, which is defined by the minimum cost of transporting the mass of one distribution to another, as detailed below. We call this diversity criterion the Wasserstein distance to a uniform distribution (WDUD). A smaller WDUD value implies that selected molecules are more diverse since the distribution of their property values is closer to being uniform.

Formally, WDUD is defined as follows. Let $v_{\max }$ and $v_{\min }$ be the maximum and minimum property values, respectively, in a given list of molecules. Suppose that $k$ molecules with property values $y_{1}, y_{2}, \cdots, y_{k}$ are selected from the list. We assign probability mass $1 / k$ to each $y_{i}$ and compute how far this discrete distribution is from a uniform distribution over $\left[v_{\min }, v_{\max }\right]$. Let $V$ and $U$ be the cumulative distribution functions of the two distributions, respectively. Defining the transportation cost from $y \in\left[v_{\min }, v_{\max }\right]$ to $y_{i}$ as $\left|y-y_{i}\right|$, the WDUD value can be computed as $\int_{v_{\min }}^{v_{\max }}|U(x)-V(x)| \mathrm{d} x{ }^{43}$ which we use for quantifying the diversity of property values $\left\{y_{1}, y_{2}, \cdots, y_{k}\right\}$ of selected molecules.

There are other possible choices of statistical distances, such as the variance or the Kullback-Leibler (KL) divergence. However, the Wasserstein distance is more suitable for measuring the diversity than the variance and the KL divergence for the 
following reasons. If we use the variance of property values of selected molecules as a diversity measure, a set of molecules with extreme property values is regarded as diverse, although this selection is biased since it ignores property values nearby the mean (see, Figure 3a). If we use the KL divergence between the property-value distribution of selected molecules and the uniform distribution, the distance structure of the support is ignored unlike WDUD, which takes the $\ell_{1}$-distance, $\left|y-y_{i}\right|$, into account. As a result, we cannot distinguish molecular sets with completely different diversities as in Figure 3b.

\section{Existing selection methods and evaluation criterion}

In the computational experiments, we use MaxMin and MaxSum as baseline methods, which greedily select molecules based on dissimilarities of molecular descriptors. We use MACCS keys and ECFP as descriptors and define the dissimilarity of those descriptors based on the Tanimoto coefficient, i.e., given the $i$-th and $j$-th descriptors, we compute the Tanimoto similarity of them and subtract it from 1 to obtain dissimilarity values $d_{i, j}$. Given dissimilarity values $d_{i, j}$, MaxSum and MaxMin operate as with the greedy algorithm; formally, MaxMin (resp. MaxSum) sequentially adds $i \in N \backslash S$ with the largest min $\operatorname{mis}_{j \in S} d_{i, j}$ (resp. $\sum_{j \in S} d_{i, j}$ ) value to $S$ whlie $|S|<k$ holds, where the first molecule $i \in N$ is set to the one with the largest $\sum_{j \neq i} d_{i, j}$ value. We denote MaxMin and MaxSum methods by MM and MS, respectively, and MACCS keys and ECFP by MK and EF, respectively, for short. We use, for example, MS-MK to represent the MaxSum method that uses MACCS-keys as descriptors.

When evaluating selection methods in the experiments, we also use MPD, an existing structure-based criterion, in addition to WDUD. Specifically, given dissimilarity values $d_{i, j}$ for all pairs in $n$ molecules, we compute an MPD value as $\frac{1}{\left(\begin{array}{c}n \\ 2\end{array}\right)} \sum_{i<j} d_{i, j}$. We define the dissimilarity values by the Tanimoto dissimilarity of MACCS keys or ECFP. Depending on the choice of descriptors, we denote the diversity criterion by MPD-MK or MPD-EF, respectively.

\section{Details of computaitonal experiments}

We conducted computational experiments with the QM9 dataset in MoleculeNet, ${ }^{50,51}$ which is a quantum mechanical dataset with labels of energetic, electronic, and thermodynamic properties computed based on the density functional theory (DFT). Each molecule in the dataset is associated with 12 property values: dipole moment in Debye (mu), isotropic polarizability in Bohr $^{3}$ (alpha), highest occupied molecular orbital energy in Hartree (HOMO), lowest unoccupied molecular orbital energy in Hartree (LUMO), gap between HOMO and LUMO in Hartree (gap), electronic spatial extent in Bohr ${ }^{2}$ (R2), zero-point vibrational energy in Hartree (ZPVE), internal energy at $0 \mathrm{~K}$ in Hartree (U0), internal energy at $298.15 \mathrm{~K}$ in Hartree (U), enthalpy at $298.15 \mathrm{~K}$ in Hartree $(\mathrm{H})$, free energy at 298.15 K in Hartree $(\mathrm{G})$, and heat capacity at $298.15 \mathrm{~K} \mathrm{in} \mathrm{cal} /(\mathrm{mol} \mathrm{K})(\mathrm{Cv})$. Following the previous work, ${ }^{36}$ we used all the 12 properties to train GNNs. The QM9 dataset contains 133885 molecules, and we randomly divided them into three datasets as is done in the previous study: ${ }^{36} 80 \%$ (107108 molecules) for training a GNN, $10 \%$ (13389 molecules) for validating prediction accuracy of the trained GNN, and 10\% (13388 molecules) for a test dataset, from which we selected molecules. Each method selected 133 molecules (1\% of the test data) from the test data. Note that when training GNNs, we did not use the test data. We thus created the situation where we select molecules whose property values are unknown in advance.

The diversity of property values of selected molecules was evaluated by computing WDUD values for each molecular property. In this evaluation, we used the above 12 properties in the QM9 dataset. However, among the 12 properties, the use of U0, U, H, and G would be inappropriate for evaluating the chemical diversity because their magnitudes depend mostly on the system size. For example, these values are more similar between acetone and acetamide, isoelectronic molecules, than between acetone and methyl-ethyl-ketone, even though most chemists would say that acetone and methyl-ethyl-ketone are both alkyl ketones and chemically more similar. Therefore, we additionally used molecular energy values divided by the number of electrons (denoted by $N_{\text {elec }}$ ) in the evaluation to weaken the system-size dependence and focus more on chemical diversity. These values for $\mathrm{U} 0, \mathrm{U}, \mathrm{H}$, and $\mathrm{G}$ are denoted by $\mathrm{U} 0 / N_{\text {elec }}, \mathrm{U} / N_{\text {elec }}, \mathrm{G} / N_{\text {elec }}$, and $\mathrm{H} / N_{\text {elec }}$, respectively. Similarly, we used variants of the two values, ZPVE and Cv, divided by $N_{\text {mode }}=3 N_{\text {atom }}-6$, where $N_{\text {atom }}$ is the number of atoms. These values for ZPVE and $\mathrm{Cv}$ are denoted by ZPVE/ $N_{\text {mode }}$ and $\mathrm{Cv} / N_{\text {mode }}$, respectively. Consequently, for evaluating molecular diversity based on WDUD values, we used 18 properties in total: the 12 properties of the QM9 dataset and the additional six properties, $\mathrm{ZPVE} / N_{\text {mode }}, \mathrm{U} 0 / N_{\text {elec }}, \mathrm{U} / N_{\text {elec }}, \mathrm{G} / N_{\text {elec }}, \mathrm{H} / N_{\text {elec }}$, and $\mathrm{Cv} / N_{\text {mode }}$.

\section{Results and discussion}

We show the results obtained by the molecular selection methods and random sampling (Random), which selects 133 molecules from the test dataset at random. We call our selection method SubMo-GNN (Submodularity-based Molecular selection with GNN-based molecular vectors). As mentioned above, MS-MK and MM-MK (MS-EF and MM-EF) stand for the existing MaxSum and MaxMin methods, respectively, that use MACCS keys (ECFP) as molecular descriptors. 


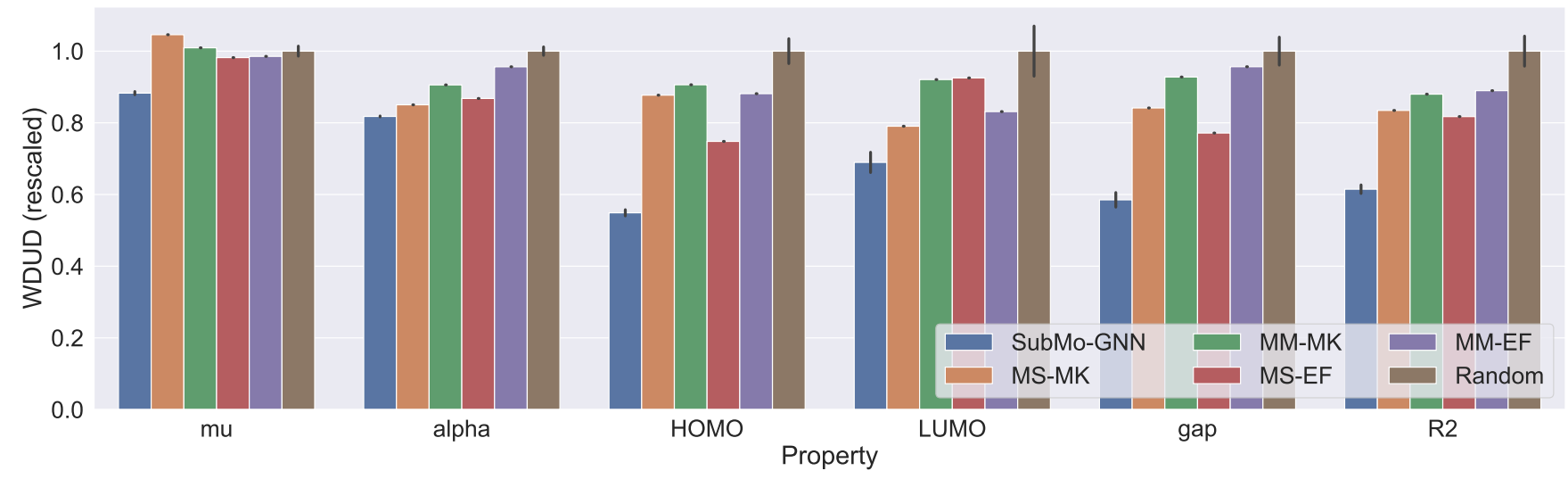

(a)

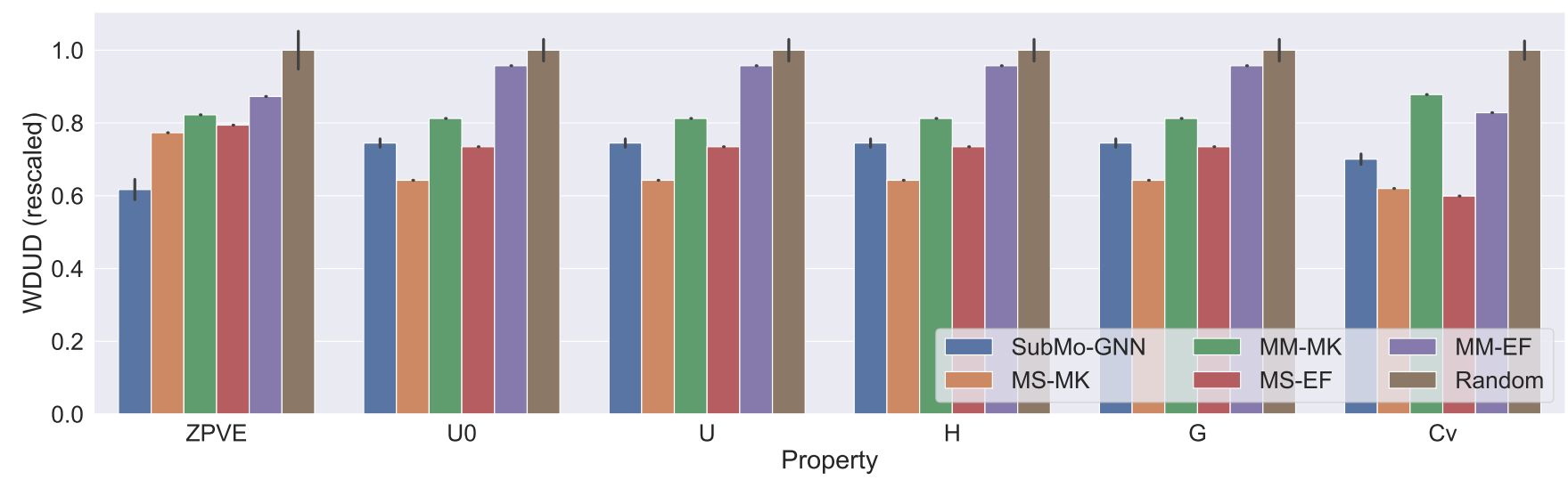

(b)

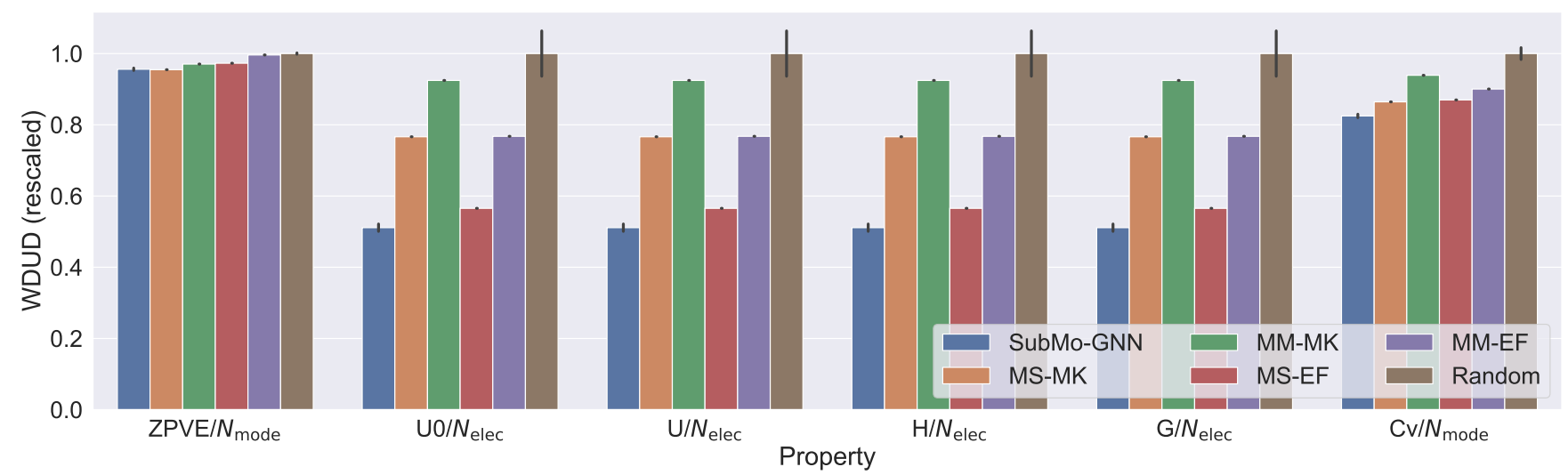

(c)

Figure 4. Bar charts of WDUD values. All the values are rescaled so that those of Random become 1. Figure 4a shows the results on the first six properties. Figure $4 \mathrm{~b}$ is the results on the six properties correlated with $N_{\text {elec }}$ or $N_{\text {mode }}$ (the WDUD values are computed with the raw property values). Figure $4 \mathrm{c}$ is a modified version of Figure $4 \mathrm{~b}$, where the property values are divided by $N_{\text {elec }}$ or $N_{\text {mode }}$ for making those values capture subtle molecular characteristics such as connectivity patterns. 
Table 1. WDUD values for each property in QM9 dataset. Since our SubMo-GNN and Random have randomness, their results are shown by means and standard deviations over 5 trials.

\begin{tabular}{|c|c|c|c|c|c|c|}
\hline & SubMo-GNN & MS-MK & MM-MK & MS-EF & MM-EF & Random \\
\hline mu (Debye) & $\mathbf{1 0 . 6 5} \pm \mathbf{0 . 0 6 0 3 3}$ & 12.62 & 12.18 & 11.85 & 11.89 & $12.06 \pm 0.1887$ \\
\hline alpha $\left(\right.$ Bohr $\left.^{3}\right)$ & $37.03 \pm 0.06616$ & 38.5 & 41.01 & 39.3 & 43.3 & $45.28 \pm 0.5972$ \\
\hline HOMO (Hartree) & $0.02786 \pm 0.0004935$ & 0.04452 & 0.04599 & 0.03797 & 0.04473 & $0.05075 \pm 0.001972$ \\
\hline LUMO (Hartree) & $\mathbf{0 . 0 2 9 0 5} \pm \mathbf{0 . 0 0 1 3 3 1}$ & 0.03331 & 0.03879 & 0.03898 & 0.03502 & $0.04213 \pm 0.003293$ \\
\hline gap (Hartree) & $\mathbf{0 . 0 3 5 4 1} \pm \mathbf{0 . 0 0 1 3 7 7}$ & 0.05092 & 0.05614 & 0.04668 & 0.05788 & $0.06051 \pm 0.002638$ \\
\hline R2 $\left(\mathrm{Bohr}^{2}\right)$ & $359.0 \pm 7.806$ & 487.3 & 513.8 & 477.3 & 519.5 & $583.9 \pm 27.37$ \\
\hline ZPVE (Hartree) & $0.0232 \pm 0.001179$ & 0.02907 & 0.03093 & 0.02986 & 0.03282 & $0.0376 \pm 0.00219$ \\
\hline U0 (Hartree) & $85.09 \pm 1.471$ & 73.39 & 92.8 & 83.92 & 109.4 & $114.2 \pm 3.789$ \\
\hline U (Hartree) & $85.09 \pm 1.471$ & 73.39 & 92.8 & 83.92 & 109.3 & $114.2 \pm 3.789$ \\
\hline H (Hartree) & $85.09 \pm 1.471$ & 73.39 & 92.8 & 83.92 & 109.3 & $114.2 \pm 3.789$ \\
\hline G (Hartree) & $85.1 \pm 1.471$ & 73.39 & 92.81 & 83.93 & 109.4 & $114.2 \pm 3.789$ \\
\hline $\mathrm{Cv}(\mathrm{cal} /(\mathrm{mol} \mathrm{K}))$ & $5.593 \pm 0.1285$ & 4.95 & 7.011 & 4.785 & 6.613 & $7.984 \pm 0.2264$ \\
\hline ZPVE $/ N_{\text {mode }}($ Hartree$)$ & $0.001533 \pm 6.145 \mathrm{e}-06$ & 0.001531 & 0.001557 & 0.001561 & 0.001598 & $0.001604 \pm 3.517 \mathrm{e}-06$ \\
\hline $\mathrm{U} 0 / N_{\text {elec }}$ (Hartree) & $\mathbf{0 . 3 8 4 8} \pm \mathbf{0 . 0 0 8 7 2 8}$ & 0.577 & 0.6961 & 0.4257 & 0.578 & $0.7529 \pm 0.05359$ \\
\hline $\mathrm{U} / N_{\text {elec }}$ (Hartree) & $0.3848 \pm 0.008728$ & 0.577 & 0.6961 & 0.4257 & 0.578 & $0.7529 \pm 0.05359$ \\
\hline $\mathrm{H} / N_{\text {elec }}$ (Hartree) & $0.3848 \pm 0.008728$ & 0.577 & 0.6961 & 0.4257 & 0.578 & $0.7529 \pm 0.05359$ \\
\hline $\mathrm{G} / N_{\text {elec }}$ (Hartree) & $0.3848 \pm \mathbf{0 . 0 0 8 7 2 8}$ & 0.577 & 0.6961 & 0.4257 & 0.578 & $0.7529 \pm 0.05359$ \\
\hline $\mathrm{Cv} / N_{\text {mode }}(\mathrm{cal} /(\mathrm{mol} \mathrm{K}))$ & $0.4681 \pm 0.003121$ & 0.4905 & 0.5328 & 0.4936 & 0.5109 & $0.5674 \pm 0.01057$ \\
\hline
\end{tabular}

\section{Property-based diversity evaluation with WDUD}

We evaluated the diversity of property values of selected molecules by the Wasserstein distance to uniform distribution (WDUD). Note that a smaller WDUD value is better since it means the distribution of selected molecules is closer to being uniform.

Table 1 shows the WDUD values attained by the six methods for the aforementioned 18 properties. Since the results of our SubMo-GNN fluctuate due to the randomness caused when training GNNs, we performed five independent runs and calculated the mean and standard deviation. The results of Random also vary from trial to trial, and thus we present the mean and standard deviation of five independent trials. Figure 4 visualizes the results in Table 1, where the WDUD values are rescaled so that those of Random become 1 for ease of comparison.

In this experiment, each method obtains a single set of molecules, for which we calculate the 18 WDUD values. Therefore, choosing a set of molecules that attains small WDUD values for some properties may result in large WDUD values for other properties. Such a choice of molecules does not meet our purpose, and it is better to balance the trade-off so that none of the 18 WDUD values become too large. A reasonable way to check whether this is achieved is to compare the results with those of Random, which selects molecules randomly according to the original distributions. If WDUD values of some properties become larger than those of Random, it is probable that the method selects molecules in a biased manner where the diversity of some properties is sacrificed for achieving small WDUD values of other properties.

We first compare the results of the selection methods with those of Random. Figure 4 shows that SubMo-GNN, MS-EF, and MM-EF attained smaller WDUD values than Random for all molecular properties. This indicates that both our method and the ECFP-based methods were able to choose diverse molecules in terms of WDUD, even though they do not explicitly minimize WDUD. Since we did not feed the test dataset when training GNNs, the results suggest that GNNs well generalized to unknown molecules and achieved diverse selection from the test dataset consisting of unknown molecules. In contrast to SubMo-GNN and the ECFP-based methods, MS-MK and MM-MK resulted in larger WDUD values in mu than Random as in Figure 4a. This suggests that MS-MK and MM-MK selected molecules in a biased manner trying to achieve small WDUD values in some properties, resulting in non-diverse selection as regards mu. In particular, MS-MK seems to have selected molecules in an attempt to achieve small WDUD values in U0, U, H, G, and Cv (see, Figure 4b) at the sacrifice of diversity in mu.

We then compare our SubMo-GNN with the existing selection methods. Compared to MaxMin-based methods (MM-MK and MM-EF), SubMo-GNN achieved smaller WDUD values for all properties. SubMo-GNN also outperformed MaxSum-based methods (MS-MK and MS-EF) for all but six properties (U0, U, H, G, Cv, and ZPVE/ $N_{\text {mode }}$ ). Note that U0, U, H, and G are related to molecular energies and their values are strongly correlated with each other; previous studies have reported that property prediction methods applied to the QM9 dataset exhibited almost the same performances as regards the four properties. ${ }^{36}$ This is consistent with our results in Figure 4b, where each method attained almost the same performance regarding the four properties. Furthermore, when the energy-related properties are divided by $N_{\text {elec }}$, MS-MK and MS-EF are 
Table 2. MPD values with MACCS keys and ECFP. For SubMo-GNN and Random, means and standard deviations over 5 trials are shown.

\begin{tabular}{lrrrrrr}
\hline & SubMo-GNN & MS-MK & MM-MK & MS-EF & MM-EF & Random \\
\hline MPD-MK & $0.8423 \pm 0.002881$ & $\mathbf{0 . 9 1 0 2}$ & 0.8592 & 0.8565 & 0.7924 & $0.7734 \pm 0.01285$ \\
MPD-EF & $0.9518 \pm 0.001709$ & 0.9558 & 0.9427 & $\mathbf{0 . 9 7 3 3}$ & 0.952 & $0.9247 \pm 0.006331$ \\
\hline
\end{tabular}

outperformed by SubMo-GNN (see the results on U0/Nelec, $\mathrm{U} / N_{\text {elec }}, \mathrm{H} / N_{\text {elec }}$, and G/ $/ N_{\text {elec }}$ in Figure $4 \mathrm{c}$ ). In view of this, the MaxSum-based methods seem to have put too much weight on the diversity of properties correlated with $N_{\text {elec }}$, which resulted in biased selections and degraded the WDUD values of mu. In summary, in terms of WDUD values, the overall performance of SubMo-GNN is better than those of the existing methods.

Figure 5 shows property-value distributions of all molecules in the dataset (blue) and molecules selected by each method (red). The horizontal and vertical axes represent property values and frequency, respectively. For ease of comparison, the histogram height is normalized to indicate the density rather than the count. We regard a set of molecules as diverse if its distribution is close to being uniform. As expected, the distribution of molecules selected by Random is close to the distribution of the original dataset. By contrast, SubMo-GNN and MS-MK selected molecules that did not appear so frequently in the dataset, particularly for $\mathrm{HOMO}, \mathrm{R} 2, \mathrm{U} 0$, and $\mathrm{U} 0 / N_{\text {elec }}$. As a result, the distributions of selected molecules became closer to being uniform than Random. Regarding the results of mu, both SubMo-GNN and MS-MK chose many molecules with near-zero mu values; this seems to be necessary for selecting diverse molecules regarding other properties than mu due to the aforementioned trade-off between properties. Nevertheless, MS-MK chose too many molecules with near-zero mu values, resulting in a biased distribution. This visually explains why the WDUD value of MS-MK in mu is larger than that of Random. Compared with MS-MK, SubMo-GNN selected more molecules with large mu values, which alleviated the bias and led to diverse selection in all properties. SubMo-GNN selected more molecules with large R2 and high HOMO values than MS-MK, and consequently SubMo-GNN's distributions were closer to being uniform. In U0, however, MS-MK selected more molecules with high U0 values than SubMo-GNN and MS-MK's distribution was closer to being uniform than SubMo-GNN. By contrast, as regards U0/ $N_{\text {elec }}$, MS-MK selected too many molecules with high $N_{\text {elec }}$ values compared with SubMo-GNN, resulting in a distribution that is farther from being uniform.

To conclude, by incorporating supervised learning of GNNs into the system of diverse molecular selection, our method can select diverse molecules regarding target molecular properties in the sense that their distributions are close to being uniform. On the other hand, if we use standard molecular descriptors (e.g., MACCS keys and ECFP) that encode only structural information of molecules, selected molecules can be biased regarding some molecular properties.

In the supplementary information, we provide additional experimental results, which digress from the main subject. We conduct an ablation study to show what if not only SubMo-GNN but also MaxSum and MaxMin use our GNN-based molecular vectors, and demonstrate that the MaxSum method with our GNN-based vector can also achieve small WDUD values, although its high performance can be brittle as detailed therein. We also take a closer look at the GNN-based vector-generation process and examine how the normalization layer affects the selection step.

\section{Structure-based diversity evaluation with MPD}

We then evaluated the diversity of the molecular selection methods in terms of molecular substructures. As a criterion for evaluating the diversity of molecular substructures, we used the mean pairwise dissimilarity (MPD), where molecular descriptors were given by MACCS keys or ECFP. We denote those criteria by MPD-MK and MPD-EF for short. A larger MPD value is better since it implies that selected molecules are more dissimilar to each other. It should be noted that MS-MK and MS-EF greedily maximize MPD-MK and MDP-EF, respectively, and thus they are inherently advantageous in this setting. MM-MK and MM-EF also explicitly maximize the diversity calculated with MACCS keys and ECFP, respectively, and thus the setting is also favorable for them. By contrast, our SubMo-GNN uses neither MACCS keys nor ECFP, and thus it has no inherent advantage as opposed to the other methods.

Table 2 shows the results. Our method and the existing methods outperformed Random in terms of both MPD-MK and MPD-EF, implying that all the selection methods successfully selected diverse molecules in terms of molecular substructures. Notably, although SubMo-GNN did not use the structure-based descriptors (MACCS keys and ECFP), it selected molecules with diverse substructures and attained higher MPD values than Random. This is achieved because when training GNNs with property prediction tasks, GNNs generate molecular vectors by taking structures of molecular graphs into account. These results and those in the previous section demonstrate that our SubMo-GNN can select diverse molecules in terms of both molecular properties and substructures.

MS-MK and MM-MK, which explicitly aim to maximize the diversity calculated with MACCS keys, achieved high 

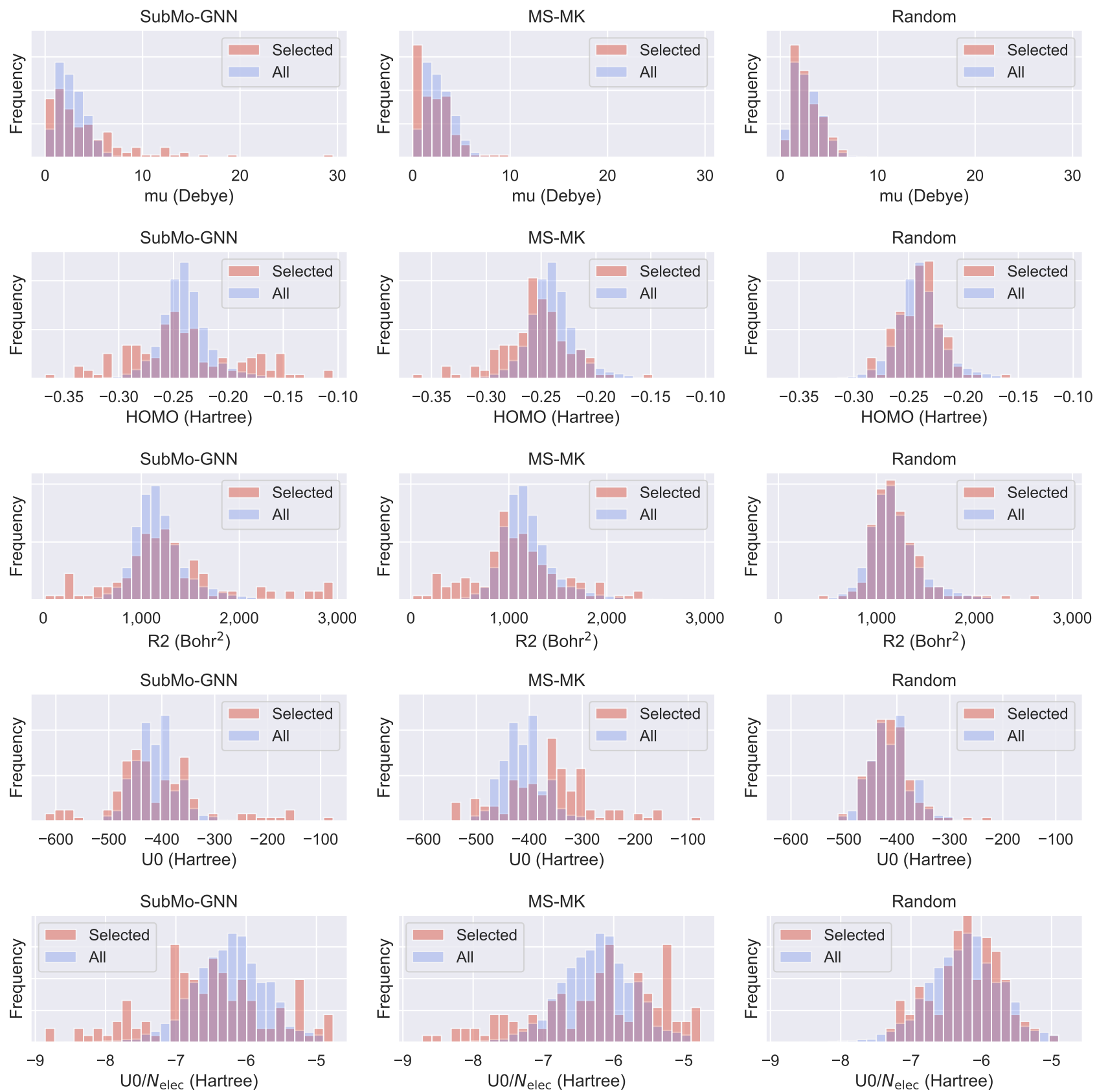

Figure 5. Property-value distributions of selected molecules (red) and all molecules in the test dataset (blue). The horizontal axis indicates property values, which are partitioned into 30 bins. We let the height show the probability density for ease of comparison. Results of the same property and method are aligned in a row and a column, respectively. 
MPD-MK values. In particular, MS-MK attained a far higher MPD-MK value than the others. This result is natural since MS-MK has the inherent advantage of greedily maximizing MPD-MK. As regards MPD-EF, all methods achieved high MPD values. Notably, SubMo-GNN delivered almost the same performance as MM-EF, which explicitly maximizes the diversity calculated with ECFP. This implies that our GNN-based molecular vectors capture structural characteristics of molecules as well as ECFP.

\section{Remark on direct minimization of WDUD}

In the above experiments, our method selected molecules based on the diversity of vectors generated by trained GNNs, which were then evaluated by WDUD (and MPD) values. Considering the evaluation criterion, one may think that it is better to select molecules by directly minimizing WDUD values instead of selecting molecular vectors. This approach, however, does not fit our goal, as discussed below.

In this study, we aim to select diverse molecules for efficiently exploring the chemical space. To this end, we utilized the power of GNNs, which can generate vectors taking both molecular structures and properties into account. As a result, our method achieved diverse selection not only in molecular properties but also in molecular structures. On the other hand, the direct minimization approach focuses only on a fraction of molecular properties used for computing WDUD values, and thus it is prone to overfitting; selected molecules may not be diverse with respect to unknown properties. In addition, the direct WDUD minimization requires property values of all candidate molecules to be known, hence its limited application scope. A possible remedy for these issues is using DFT or other machine learning methods to predict unknown property values. This, however, is computationally more costly than our method. Moreover, even if unknown property values are successfully predicted, minimizing WDUD values is also a computationally hard task for which no efficient algorithm is available to the best of our knowledge. In a nutshell, although the direct minimization approach may improve WDUD values, it is not a reasonable way to select diverse molecules for efficiently exploring the chemical space.

\section{Conclusion}

We addressed the problem of selecting diverse molecules for facilitating chemical space exploration. Our method consists of two steps: construction of molecular vectors using the GNN and selection of molecules via maximizing submodular functions defined with molecular vectors. Owing to the use of GNNs trained with property prediction tasks, we can take both molecular structures and properties into account for selecting diverse molecules. Moreover, the submodular function maximization framework enables the greedy algorithm to return subsets of molecules, which are mathematically guaranteed to be nearly optimal. We also introduced a new evaluation criterion, the Wasserstein distance to uniform distributions (WDUD), to measure the diversity of sets of molecules based on property values. Computational experiments on the QM9 dataset showed that our method could successfully select diverse molecules as regards property values. Regarding the diversity of molecular structures, it performed comparably to the existing structure-based methods (MaxSum and MaxMin with MACCS keys and ECFP). Our diverse selection method helps researchers efficiently explore the chemical space, which will bring great advances in searching for novel chemical compounds and reactions.

\section{Data availability}

Source codes of our method are available at https://github. com/tomotomonakanaka/SUBMO.git, which were implemented in Python 3.7.10. We converted SMILES into MACCS key, ECFP, and molecular graphs by using RDKit 2018.09.1, which is available at https: / /www.rdkit.org/. The QM9 dataset was downloaded from Xiong's GitHub repository (https: / / github. com/OpenDrugAI/AttentiveFP). GNNs were implemented using PyTorch 1.8.0, ${ }^{52}$ DGL 0.5.3, ${ }^{53}$ and DGL-LifeSci 0.2.6 (available at https://github.com/awslabs/dgl-lifesci). We trained Attentive FP with the following hyperparameters: radius $=2, \mathrm{~T}=2$, fingerprint dimension $=300$, dropout $=0.5$, weight decay $=0$, learning rate $=0.0004$, and epoch $=300$, where radius and $\mathrm{T}$ are the number of times the hidden states are updated in the message passing phase and the readout phase, respectively.

\section{References}

1. Kirkpatrick, P. \& Ellis, C. Chemical space. Nature 432, 823-823 (2004).

2. Reymond, J.-L., Ruddigkeit, L., Blum, L. \& van Deursen, R. The enumeration of chemical space. WIREs Comput. Mol. Sci. 2, 717-733 (2012).

3. Reymond, J.-L. \& Awale, M. Exploring chemical space for drug discovery using the chemical universe database. ACS Chem. Neurosci. 3, 649-657 (2012).

4. Reymond, J.-L. The chemical space project. Acc. Chem. Res. 48, 722-730 (2015). 
5. Alain-Dominique, G. Diversity in medicinal chemistry space. Curr. Top. Med. Chem. 6, 3-18 (2006).

6. Bohacek, R. S., McMartin, C. \& Guida, W. C. The art and practice of structure-based drug design: A molecular modeling perspective. Med. Res. Rev. 16, 3-50 (1996).

7. Ertl, P. Cheminformatics analysis of organic substituents: Identification of the most common substituents, calculation of substituent properties, and automatic identification of drug-like bioisosteric groups. J. Chem. Inf. Comput. Sci. 43, 374-380 (2003).

8. Hamill, K. A., Nelson, R. D., Vander Stouw, G. G. \& Stobaugh, R. E. Chemical abstracts service chemical registry system. 10. registration of substances from pre-1965 indexes of chemical abstracts. J. Chem. Inf. Comput. Sci. 28, 175-179 (1988).

9. American Chemical Society. CAS - chemical abstracts service - database counter. http://web.cas.org/cgi-bin/regreport.pl (accessed Jan 31, 2021).

10. Kim, S. et al. PubChem 2019 update: Improved access to chemical data. Nucleic Acids Res. 47, D1102-D1109 (2019).

11. Irwin, J. J. \& Shoichet, B. K. ZINC-a free database of commercially available compounds for virtual screening. J. Chem. Inf. Model. 45, 177-182 (2005).

12. Takeda, S., Kaneko, H. \& Funatsu, K. Chemical-space-based de novo design method to generate drug-like molecules. $J$. Chem. Inf. Model. 56, 1885-1893 (2016).

13. Sanchez-Lengeling, B. \& Aspuru-Guzik, A. Inverse molecular design using machine learning: Generative models for matter engineering. Science 361, 360-365 (2018).

14. Gómez-Bombarelli, R. et al. Design of efficient molecular organic light-emitting diodes by a high-throughput virtual screening and experimental approach. Nat. Mater. 15, 1120-1127 (2016).

15. Ahneman, D. T., Estrada, J. G., Lin, S., Dreher, S. D. \& Doyle, A. G. Predicting reaction performance in c-n cross-coupling using machine learning. Science 360, 186-190 (2018).

16. Zahrt, A. F. et al. Prediction of higher-selectivity catalysts by computer-driven workflow and machine learning. Science 363, eaau5631 (2019).

17. Gillet, V. J. Diversity selection algorithms. WIREs Comput. Mol. Sci. 1, 580-589 (2011).

18. Lajiness, M. \& Watson, I. Dissimilarity-based approaches to compound acquisition. Curr. Opin. Chem. Biol. 12, 366-371 (2008).

19. Rognan, D. The impact of in silico screening in the discovery of novel and safer drug candidates. Pharmacol. Ther. 175, 47-66 (2017).

20. Gorgulla, C. et al. An open-source drug discovery platform enables ultra-large virtual screens. Nature 580, 663-668 (2020).

21. Grygorenko, O. O., Volochnyuk, D. M., Ryabukhin, S. V. \& Judd, D. B. The symbiotic relationship between drug discovery and organic chemistry. Chem. Eur. J. 26, 1196-1237 (2020).

22. Maldonado, A. G., Doucet, J. P., Petitjean, M. \& Fan, B.-T. Molecular similarity and diversity in chemoinformatics: From theory to applications. Mol. Divers. 10, 39-79 (2006).

23. Rogers, D. \& Hahn, M. Extended-connectivity fingerprints. J. Chem. Inf. Model. 50, 742-754 (2010).

24. Symyx Technologies Inc. Maccs keys.

25. Daylight Chemical Information Systems, Inc. Daylight fingerprints.

26. Tanimoto, T. T. An Elementary Mathematical Theory of Classification and Prediction (International Business Machines Corporation, New York, 1958).

27. Fukutani, T., Miyazawa, K., Iwata, S. \& Satoh, H. G-RMSD: Root mean square deviation based method for threedimensional molecular similarity determination. Bull. Chem. Soc. Jpn. 94, 655-665 (2021).

28. Lajiness, M. S. Molecular similarity-based methods for selecting compounds for screening, 299-316 (Nova Science Publishers, Inc., USA, 1990).

29. Holliday, J. D., Ranade, S. S. \& Willett, P. A fast algorithm for selecting sets of dissimilar molecules from large chemical databases. Quant. Struct.-Act. Relat. 14, 501-506 (1995).

30. Snarey, M., Terrett, N. K., Willett, P. \& Wilton, D. J. Comparison of algorithms for dissimilarity-based compound selection. J. Mol. Graph. Model. 15, 372-385 (1997). 
31. Agrafiotis, D. K. \& Lobanov, V. S. An efficient implementation of distance-based diversity measures based on $k$ - $d$ trees. $J$. Chem. Inf. Comput. Sci. 39, 51-58 (1999).

32. Terayama, K. et al. Pushing property limits in materials discovery via boundless objective-free exploration. Chem. Sci. 11, 5959-5968 (2020).

33. Duvenaud, D. K. et al. Convolutional networks on graphs for learning molecular fingerprints. In Advances in Neural Information Processing Systems, vol. 28, 2224-2232 (2015).

34. Gilmer, J., Schoenholz, S. S., Riley, P. F., Vinyals, O. \& Dahl, G. E. Neural message passing for quantum chemistry. In Proceedings of the 34th International Conference on Machine Learning, vol. 70, 1263-1272 (2017).

35. Schütt, K. T. et al. Schnet: A continuous-filter convolutional neural network for modeling quantum interactions. In Advances in Neural Information Processing Systems, vol. 30, 991-1001 (2017).

36. Xiong, Z. et al. Pushing the boundaries of molecular representation for drug discovery with the graph attention mechanism. J. Med. Chem. 63, 8749-8760 (2020).

37. Rahaman, O. \& Gagliardi, A. Deep learning total energies and orbital energies of large organic molecules using hybridization of molecular fingerprints. J. Chem. Inf. Model. 60, 5971-5983 (2020).

38. Hwang, D. et al. Comprehensive study on molecular supervised learning with graph neural networks. J. Chem. Inf. Model. 60, 5936-5945 (2020).

39. Nemhauser, G. L., Wolsey, L. A. \& Fisher, M. L. An analysis of approximations for maximizing submodular set functions-I. Math. Program. 14, 265-294 (1978).

40. Krause, A. \& Golovin, D. Submodular function maximization, 71-104 (Cambridge University Press, 2014).

41. Zhou, L. et al. Graph neural networks for decentralized multi-robot submodular action selection. arXiv preprint arXiv:2105.08601 (2021).

42. Vaserstein, L. N. Markov processes over denumerable products of spaces, describing large systems of automata. Probl. Peredachi Inf. 5, 64-72 (1969).

43. Peyré, G. \& Cuturi, M. Computational optimal transport: With applications to data science. Found. Trends Mach. Learn. 11, 355-607 (2019).

44. Lin, C.-Y. ROUGE: A package for automatic evaluation of summaries. In Text Summarization Branches Out, 74-81 (Association for Computational Linguistics, 2004).

45. Lin, H. \& Bilmes, J. A class of submodular functions for document summarization. In Proceedings of the 49th Annual Meeting of the Association for Computational Linguistics: Human Language Technologies, 510-520 (ACL, 2011).

46. Cornuejols, G., Fisher, M. L. \& Nemhauser, G. L. Location of bank accounts to optimize float: An analytic study of exact and approximate algorithms. Manag. Sci. 23, 789-810 (1977).

47. Bai, W., Bilmes, J. \& Noble, W. S. Submodular generalized matching for peptide identification in tandem mass spectrometry. IEEE/ACM Trans. Comput. Biol. Bioinform. 16, 1168-1181 (2019).

48. Kulesza, A. \& Taskar, B. Determinantal Point Processes for Machine Learning (Now Publishers Inc., 2012).

49. Chen, L., Zhang, G. \& Zhou, E. Fast greedy map inference for determinantal point process to improve recommendation diversity. In Advances in Neural Information Processing Systems, vol. 31, 5627-5638 (Curran Associates, Inc., 2018).

50. Ramakrishnan, R., Dral, P. O., Rupp, M. \& von Lilienfeld, O. A. Quantum chemistry structures and properties of 134 kilo molecules. Sci. Data 1, 140022-140029 (2014).

51. Wu, Z. et al. MoleculeNet: A benchmark for molecular machine learning. Chem. Sci. 9, 513-530 (2018).

52. Paszke, A. et al. Pytorch: An imperative style, high-performance deep learning library. In Advances in Neural Information Processing Systems, vol. 32, 8026-8037 (2019).

53. Wang, M. et al. Deep graph library: A graph-centric, highly-performant package for graph neural networks. arXiv preprint arXiv:1909.01315 (2019).

\section{Acknowledgements}

This work was supported by the JST via ERATO grant JPMJER1903. Support was also provided by the Institute for Chemical Reaction Design and Discovery (ICReDD), which was established by the World Premier International Research Initiative (WPI), MEXT, Japan. 


\section{Author contributions}

S.S., K.F., and Y.H. formulated the problem and developed a basic method. T.N. made substantial contributions to conceptual design and conducted computational experiments with extensive help from S.S., K.F., and Y.H. T.N., S.S., K.F., and Y.H. analyzed the computed results. S.M. and S.I. improved the analysis of the results. All authors reviewed the manuscript.

\section{Competing interests}

The authors declare no competing interests.

\section{Additional information}

Correspondence and requests for materials should be addressed to S.S., K.F., and Y.H. 


\section{Supplementary Files}

This is a list of supplementary files associated with this preprint. Click to download.

- supplementary.pdf 